\title{
Inequalities of uncertain set with its applications
}

\author{
Yufu Ning ${ }^{1,2^{*}}$ and Zhiyong Huang ${ }^{3}$
}

${ }^{*}$ Correspondence:

ningyufuu@163.com

'School of Information Engineering,

Shandong Youth University of

Political Science, Jinan, 250103,

China

${ }^{2}$ Key Laboratory of Information

Security and Intelligent Control in

Universities of Shandong, Jinan,

250103, China

Full list of author information is

available at the end of the article

\begin{abstract}
An uncertain set, as a generalization of the uncertain variable, is a set-valued function on an uncertainty space. It provides theoretical foundations for uncertain inference and uncertain logic. This paper aims at providing some inequalities in the framework of uncertain set theory, including the Markov inequality, the Chebyshev inequality, the Jensen inequality, the Hölder inequality, and the Minkowski inequality. In addition, this paper applies these inequalities to the area of incomplete uncertain knowledge representation.
\end{abstract}

Keywords: uncertain set; membership function; inequality; uncertainty theory

\section{Introduction}

Probability theory, since it was founded by Kolmogorov in 1933 based on normality, nonnegativity, and countable additivity axioms, has been widely used to model the indeterminacy phenomena. A premise of applying probability is that the obtained probability distribution is close enough to the real frequency, which is ignored by many researchers. Usually, we need many samples to obtain the probability distribution via statistics, but in real life we sometimes have no sample due to economical or technological reasons. In this case, we have to invite some experts to evaluate the belief degree that a possible event occurs. For example, the bearing capacity of a bridge generally is obtained not from repeated trials but from the belief degree of bridge engineers.

The belief degree was once treated as probability distribution of a random variable, which led to some counterintuitive results. Interested readers may refer to Liu [1] for an example. In order to deal with belief degree, Zadeh [2] proposed the concept of a fuzzy set via the membership function in 1965, and Zadeh [3] proposed possibility theory in 1978 as a theoretical foundation of the fuzzy set. Except for Zadeh's possibility theory, a theory of imprecise probability was introduced by Dempster [4] in 1967, and further elaborated by Shafer [5] in 1976. Essentially, imprecise probability is a set-valued mapping on a probability space. In addition, in order to deal with the belief degree, a prospect theory was proposed by Kahneman and Tversky [6] in 1979, and a rough set theory was founded by Pawlak [7] in 1982.

In order to study the belief degree, an uncertainty theory was founded by Liu [8] in 2007 and revised by Liu [9] in 2010 based on normality, duality, subadditivity, and product axioms. In order to indicate the belief degree, concepts of uncertain measure and uncertainty space were proposed. Then Gao [10] studied the property of continuous uncertain measure. Similar to random variable, uncertain variable was defined as a measurable function

C2014 Ning and Huang; licensee Springer. This is an Open Access article distributed under the terms of the Creative Commons Attribution License (http://creativecommons.org/licenses/by/2.0), which permits unrestricted use, distribution, and reproduction in any medium, provided the original work is properly cited. 
on the uncertainty space. After that, You [11] studied the convergence of a sequence of uncertain variables. Liu and Ha [12] proved the linearity of the expected value operator of uncertain variables, and Chen and Dai [13] proposed a maximum entropy principle for uncertain variables.

In 2010, Liu [14] proposed a concept of an uncertain set as a generalization of the uncertain variable. Meanwhile, he defined a membership function to describe an uncertain set, and he gave the concept of the expected value to model the size of an uncertain set. In order to derive consequences from human knowledge, Liu [14] proposed uncertain inference via uncertain set theory. After that, Gao et al. [15] extended the inference rule to the case with multiple antecedents and multiple if-then rules. In addition, Liu [14] presented the concepts of uncertain system and uncertain inference controller. Then Peng and Chen [16] proved that an uncertain system is a universal approximator. As an application, Gao [17] balanced an inverted pendulum by using the uncertain inference control. Except for uncertain inference, the uncertain set has also been used to uncertain logic (Liu [18]) as a tool to calculate the truth value of an uncertain proposition.

In 2012, Liu [19] recast uncertain set theory. He redefined the concept of membership function and provided the operational law of uncertain sets via inverse membership functions. Meanwhile, Liu proposed the concept of entropy to describe the divergence of an uncertain set. After that, Yao [20] proved that the entropy operator meets the requirement of positive linearity. As an extension, Wang and $\mathrm{Ha}$ [21] proposed the quadratic entropy for an uncertain set. In this paper, we will give some inequalities of uncertain set as well as their applications. The rest of this paper is structured as follows. The next section is intended to introduce some concepts in uncertain set theory. Then some inequalities involving a single uncertain set are given in Section 3, and some inequalities involving multiple uncertain sets are given in Section 4. After that, we apply the inequalities to the area of representing incomplete knowledge in Section 5. At last, some remarks are made in Section 6.

\section{Preliminary}

Uncertainty theory is a branch of mathematics based on normality, duality, subadditivity, and product axioms. So far, it has brought about many branches such as uncertain programming (Liu [22]), uncertain risk analysis (Liu [1]), uncertain process (Liu [23]), uncertain differential equation (Liu [23], Yao [24]), uncertain logic (Liu [18]), uncertain finance (Liu [25], Yao [26]), and uncertain inference control (Liu [14], Gao [17]). In this section, we will introduce some useful definitions as regards the uncertain set.

Definition 1 (Liu [8]) Let $\Gamma$ be a nonempty set, and $\mathcal{L}$ be a $\sigma$-algebra on $\Gamma$. A set function $\mathcal{M}$ is called an uncertain measure if it satisfies the following axioms.

Axiom 1: (Normality) $\mathcal{M}\{\Gamma\}=1$;

Axiom 2: (Duality) $\mathcal{N}\{\Lambda\}+\mathcal{N}\left\{\Lambda^{c}\right\}=1$ for any $\Lambda \in \mathcal{L}$;

Axiom 3: (Subadditivity) For every sequence of $\left\{\Lambda_{i}\right\} \in \mathcal{L}$, we have

$$
\mathcal{M}\left\{\bigcup_{i=1}^{\infty} \Lambda_{i}\right\} \leq \sum_{i=1}^{\infty} \mathcal{M}\left\{\Lambda_{i}\right\} .
$$

In this case, the triple $(\Gamma, \mathcal{L}, \mathcal{M})$ is called an uncertainty space. 
Besides, an axiom called product axiom was given by Liu [22] for the operation of uncertain sets in 2009.

Axiom 4: (Product Axiom) Let $\left(\Gamma_{k}, \mathcal{L}_{k}, \mathcal{M}_{k}\right)$ be uncertainty spaces for $k=1,2, \ldots$.

Then the product uncertain measure $\mathcal{M}$ is an uncertain measure satisfying

$$
\mathcal{M}\left\{\prod_{i=1}^{\infty} \Lambda_{k}\right\}=\bigwedge_{k=1}^{\infty} \mathcal{M}_{k}\left\{\Lambda_{k}\right\}
$$

where $\Lambda_{k}$ are arbitrarily chosen events from $\mathcal{L}_{k}$ for $k=1,2, \ldots$, respectively.

Definition 2 (Liu [14]) An uncertain set is a measurable function $\xi$ from an uncertainty space $(\Gamma, \mathcal{L}, \mathcal{N})$ to a collection of sets of real numbers, i.e., for any Borel set $B$ of real numbers, the following two sets:

$$
\{\xi \subset B\}=\{\gamma \in \Gamma \mid \xi(\gamma) \subset B\}, \quad\{B \subset \xi\}=\{\gamma \in \Gamma \mid B \subset \xi(\gamma)\}
$$

are events.

Let $\xi$ and $\eta$ be two uncertain sets on an uncertainty space $(\Gamma, \mathcal{L}, \mathcal{M})$. Then the union $\xi \cup \eta$ of uncertain sets $\xi$ and $\eta$ is defined by

$$
(\xi \cup \eta)(\gamma)=\xi(\gamma) \cup \eta(\gamma), \quad \forall \gamma \in \Gamma .
$$

The intersection $\xi \cap \eta$ of uncertain sets $\xi$ and $\eta$ is defined by

$$
(\xi \cap \eta)(\gamma)=\xi(\gamma) \cap \eta(\gamma), \quad \forall \gamma \in \Gamma .
$$

The complement $\xi^{c}$ of the uncertain set $\xi$ is defined by

$$
\xi^{c}(\gamma)=(\xi(\gamma))^{c}, \quad \forall \gamma \in \Gamma .
$$

They are all uncertain sets.

Definition 3 (Liu [19]) An uncertain set $\xi$ is said to have a membership function $\mu$ if the equations

$$
\mathcal{M}\{B \subset \xi\}=\inf _{x \in B} \mu(x), \quad \mathcal{M}\{\xi \subset B\}=1-\sup _{x \in B^{c}} \mu(x)
$$

hold for any Borel set $B$ of real numbers.

If an uncertain set $\xi$ has a membership function $\mu$, then we have $\mu(x)=\mathcal{M}\{x \in \xi\}$. Liu [18] proved that a real-valued function $\mu$ is a membership function if and only if $0 \leq \mu(x) \leq 1$.

Example 1 Take the uncertainty space $\{\Gamma, \mathcal{L}, \mathcal{M}\}$ to be $[0,1]$ with $\mathcal{M}\{[0, \gamma]\}=\gamma$ for each $\gamma \in[0,1]$. Then the uncertain set

$$
\xi(\gamma)=[0, \sqrt{1-\gamma}]
$$


has a membership function

$$
\mu(x)= \begin{cases}1-x^{2}, & \text { if } x \in[0,1], \\ 0, & \text { otherwise }\end{cases}
$$

In order to get the membership function of an uncertain set, Liu [19] designed a questionnaire for collecting expert's experimental data, and he employed the least square method to deduce the membership function. First we get an expert's experimental data

$$
\left(x_{1}, \alpha_{1}\right), \quad\left(x_{2}, \alpha_{2}\right), \quad \ldots, \quad\left(x_{n}, \alpha_{n}\right)
$$

by asking the expert some questions like

'How likely does $x_{i}$ belong to $\xi$ ?'

Then we calculate

$$
\min _{\theta} \sum_{i=1}^{n}\left(\mu(x \mid \theta)-\alpha_{i}\right)^{2}
$$

where $\mu(x \mid \theta)$ is a membership function with an unknown parameter $\theta$.

Definition 4 (Liu [19]) Let $\xi$ be an uncertain set with a membership function $\mu$. Then the set-valued function

$$
\mu^{-1}(\alpha)=\{x \in \Re \mid \mu(x) \geq \alpha\}, \quad \forall \alpha \in[0,1]
$$

is called the inverse membership function of $\xi$.

A membership function is said to be regular if there exists a point $x_{0}$ such that $\mu\left(x_{0}\right)=1$ and $\mu(x)$ is unimodal about the point $x_{0}$. If $\mu$ is a regular membership function, then the function $\mu_{l}^{-1}(\alpha)=\inf \mu^{-1}(\alpha)$ is called the left inverse membership function, and the function $\mu_{r}^{-1}(\alpha)=\sup \mu^{-1}(\alpha)$ is called the right inverse membership function. Note that $\mu_{l}^{-1}(\alpha)$ is increasing and $\mu_{r}^{-1}(\alpha)$ is decreasing with respect to $\alpha$.

Example 2 The word 'young' may be regarded as an uncertain set $\xi$ with a membership function

$$
\mu(x)= \begin{cases}(x-15) / 5, & \text { if } 15 \leq x \leq 20 \\ 1, & \text { if } 20<x<30 \\ (35-x) / 5, & \text { if } 30 \leq x \leq 35 \\ 0, & \text { otherwise }\end{cases}
$$

In this case, the inverse membership function of $\xi$ is

$$
\mu^{-1}(\alpha)=[15+5 \alpha, 35-5 \alpha]
$$


and the left and right inverse membership functions of $\xi$ are

$$
\mu_{l}^{-1}(\alpha)=15+5 \alpha, \quad \mu_{r}^{-1}(\alpha)=35-5 \alpha,
$$

respectively.

The inverse membership function plays an important role in the operation of independent uncertain sets.

Definition 5 (Liu [27]) The uncertain sets $\xi_{1}, \xi_{2}, \ldots, \xi_{n}$ are said to be independent if for any Borel sets $B_{1}, B_{2}, \ldots, B_{n}$, we have

$$
\mathcal{M}\left\{\bigcap_{i=1}^{n}\left(\xi_{i}^{*} \subset B_{i}\right)\right\}=\bigwedge_{i=1}^{n} \mathcal{M}\left\{\xi_{i}^{*} \in B_{i}\right\}, \quad \mathcal{M}\left\{\bigcup_{i=1}^{n}\left(\xi_{i}^{*} \subset B_{i}\right)\right\}=\bigvee_{i=1}^{n} \mathcal{M}\left\{\xi_{i}^{*} \in B_{i}\right\},
$$

where $\xi_{i}^{*}$ are arbitrarily chosen from $\left\{\xi_{i}, \xi_{i}^{c}\right\}, i=1,2, \ldots, n$, respectively.

Theorem 1 (Liu [19]) Let $\xi$ and $\eta$ be independent uncertain sets with regular membership functions $\mu$ and $v$, respectively. If $f(x, y)$ is monotone increasing with respect to $x$ and $y$, then $\tau=f(\xi, \eta)$ is an uncertain set with a regular membership function $\lambda$ where

$$
\lambda_{l}^{-1}(\alpha)=f\left(\mu_{l}^{-1}(\alpha), v_{l}^{-1}(\alpha)\right), \quad \lambda_{r}^{-1}(\alpha)=f\left(\mu_{r}^{-1}(\alpha), v_{r}^{-1}(\alpha)\right) .
$$

Definition 6 (Liu [14]) Let $\xi$ be a nonempty uncertain set. Then the expected value of $\xi$ is defined by

$$
E[\xi]=\int_{0}^{+\infty} \mathcal{M}\{\xi \geq x\} \mathrm{d} x-\int_{-\infty}^{0} \mathcal{M}\{\xi \leq x\} \mathrm{d} x
$$

provided that at least one of the two integral is finite.

When the uncertain set $\xi$ has a regular membership function $\mu(x)$ with $\mu\left(x_{0}\right)=1$, Liu [19] proved that

$$
E[\xi]=x_{0}+\frac{1}{2} \int_{x_{0}}^{+\infty} \mu(x) \mathrm{d} x-\frac{1}{2} \int_{-\infty}^{x_{0}} \mu(x) \mathrm{d} x=\frac{1}{2} \int_{0}^{1}\left(\mu_{l}^{-1}(\alpha)+\mu_{r}^{-1}(\alpha)\right) \mathrm{d} \alpha .
$$

In addition, for independent uncertain sets $\xi$ and $\eta$, we have

$$
E[a \xi+b \eta]=a E[\xi]+b E[\eta]
$$

for any real numbers $a$ and $b$.

Definition 7 (Liu [14]) Let $\xi$ be an uncertain set with finite expected value $e$. Then the variance of $\xi$ is defined by

$$
V[\xi]=E\left[(\xi-e)^{2}\right] .
$$




\section{Inequalities of single uncertain set}

Inequalities play an important role in estimating the range of a variable. The Markov inequality, the Chebyshev inequality, and the Jensen inequality have been introduced to probability theory and uncertainty theory, and they have found many applications. In this section, we will consider the Markov inequality, the Chebyshev inequality, and the Jensen inequality in the framework of uncertain set theory.

Theorem 2 Let $\xi$ be an uncertain set, and $f$ be a nonnegative function. If $f$ is even and increasing on $[0,+\infty)$, then for any given number $t>0$, we have

$$
\mathcal{M}\{|\xi| \geq t\} \leq \frac{E[f(\xi)]}{f(t)}
$$

Proof Note that $\mathcal{M}\left\{|\xi| \geq f^{-1}(r)\right\}$ is a decreasing function of $r$ on $[0,+\infty)$. It follows from the nonnegativity of $f$ that

$$
\begin{aligned}
E[f(\xi)] & =\int_{0}^{+\infty} \mathcal{M}\{f(\xi) \geq r\} \mathrm{d} r=\int_{0}^{+\infty} \mathcal{M}\left\{|\xi| \geq f^{-1}(r)\right\} \mathrm{d} r \\
& \geq \int_{0}^{f(t)} \mathcal{M}\left\{|\xi| \geq f^{-1}(r)\right\} \mathrm{d} r \geq \mathcal{M}\left\{|\xi| \geq f^{-1}(f(t))\right\} \int_{0}^{f(t)} \mathrm{d} r \\
& =\mathcal{M}\{|\xi| \geq t\} f(t) .
\end{aligned}
$$

The inequality is thus verified.

Theorem 3 (Markov inequality) Let $\xi$ be an uncertain set. Then for any given numbers $t>0$ and $p>0$, we have

$$
\mathcal{M}\{|\xi| \geq t\} \leq \frac{E\left[|\xi|^{p}\right]}{t^{p}}
$$

Proof Take $f(x)=|x|^{p}$ in Theorem 2, and this inequality follows immediately.

Theorem 4 (Chebyshev inequality) Let $\xi$ be an uncertain set with a finite expected value $E[\xi]$. Then for any given number $t>0$, we have

$$
\mathcal{M}\{|\xi-E[\xi]| \geq t\} \leq \frac{V[\xi]}{t^{2}}
$$

Proof Replace the uncertain set $\xi$ with $\xi-E[\xi]$, and take $f(x)=|x|^{2}$ in Theorem 2. Then the inequality follows immediately.

Theorem 5 (Jensen inequality) Let $\xi$ be an uncertain set with regular membership function, and $f: \Re \rightarrow \Re$ be a convex function. If $E[\xi]$ and $E[f(\xi)]$ are finite, then

$$
f(E[\xi]) \leq E[f(\xi)]
$$

Especially, when $f(x)=|x|^{p}$ and $p \geq 1$, we have $|E[\xi]|^{p} \leq E\left[|\xi|^{p}\right]$. 
Proof Since $f$ is a convex function, for each $x$ and $y$, there exists a number $k$ such that $f(x)-f(y) \geq k(x-y)$. Let $\mu$ denote the membership function of $\xi$. Taking $x=\mu_{l}^{-1}(\alpha)$ and $y=E[\xi]$, we have

$$
f\left(\mu_{l}^{-1}(\alpha)\right)-f(E[\xi]) \geq k\left(\mu_{l}^{-1}(\alpha)-E[\xi]\right) .
$$

Taking $x=\mu_{r}^{-1}(\alpha)$ and $y=E[\xi]$, we have

$$
f\left(\mu_{r}^{-1}(\alpha)\right)-f(E[\xi]) \geq k\left(\mu_{r}^{-1}(\alpha)-E[\xi]\right) .
$$

By the inequalities (1) and (2), we have

$$
\frac{1}{2}\left(f\left(\mu_{l}^{-1}(\alpha)\right)+f\left(\mu_{r}^{-1}(\alpha)\right)\right)-f(E[\xi]) \geq \frac{k}{2}\left(\mu_{l}^{-1}(\alpha)+\mu_{r}^{-1}(\alpha)\right)-k E[\xi] .
$$

Taking integration with respect to $\alpha$ on $[0,1]$, we have

$$
E[f(\xi)]-f(E[\xi]) \geq k E[\xi]-k E[\xi]=0 .
$$

The inequality is thus verified.

\section{Inequalities of multiple uncertain sets}

In this section, we will propose some inequalities with multiple uncertain sets in the framework of uncertain set theory.

Theorem 6 (Hölder inequality) Let $p$ and $q$ be positive numbers such that $1 / p+1 / q=1$, and let $\xi$ and $\eta$ be independent uncertain sets with regular membership functions. Then we have

$$
E[|\xi \eta|] \leq \sqrt[p]{E\left[|\xi|^{p}\right]} \sqrt[q]{E\left[|\eta|^{q}\right]}
$$

Proof Note that $f(x, y)=\sqrt[p]{x} \sqrt[q]{y}$ is a concave function on $\{(x, y) \mid x \geq 0, y \geq 0\}$. Thus for any point $\left(x_{0}, y_{0}\right)$ with $x_{0}>0$ and $y_{0}>0$, there exist two real numbers $a$ and $b$ such that

$$
f(x, y)-f\left(x_{0}, y_{0}\right) \leq a\left(x-x_{0}\right)+b\left(y-y_{0}\right), \quad \forall x \geq 0, y \geq 0 .
$$

Let $\mu$ and $v$ denote the regular membership functions of uncertain sets $\xi$ and $\eta$, respectively. Taking $x_{0}=E\left[|\xi|^{p}\right], y_{0}=E\left[|\eta|^{q}\right], x=\left(\mu_{l}^{-1}(\alpha)\right)^{p}$ and $y=\left(v_{l}^{-1}(\alpha)\right)^{q}$, we have

$$
\begin{aligned}
& f\left(\left(\mu_{l}^{-1}(\alpha)\right)^{p},\left(v_{l}^{-1}(\alpha)\right)^{q}\right)-f\left(E\left[|\xi|^{p}\right], E\left[|\eta|^{q}\right]\right) \\
& \quad \leq a\left(\left(\mu_{l}^{-1}(\alpha)\right)^{p}-E\left[|\xi|^{p}\right]\right)+b\left(\left(v_{l}^{-1}(\alpha)\right)^{q}-E\left[|\eta|^{q}\right]\right)
\end{aligned}
$$

for all $\alpha \in(0,1)$. Taking $x_{0}=E\left[|\xi|^{p}\right], y_{0}=E\left[|\eta|^{q}\right], x=\left(\mu_{r}^{-1}(\alpha)\right)^{p}$ and $y=\left(v_{r}^{-1}(\alpha)\right)^{q}$, we have

$$
\begin{aligned}
& f\left(\left(\mu_{r}^{-1}(\alpha)\right)^{p},\left(v_{r}^{-1}(\alpha)\right)^{q}\right)-f\left(E\left[|\xi|^{p}\right], E\left[|\eta|^{q}\right]\right) \\
& \quad \leq a\left(\left(\mu_{r}^{-1}(\alpha)\right)^{p}-E\left[|\xi|^{p}\right]\right)+b\left(\left(v_{r}^{-1}(\alpha)\right)^{q}-E\left[|\eta|^{q}\right]\right)
\end{aligned}
$$


for all $\alpha \in(0,1)$. By the inequalities (3) and (4), we have

$$
\begin{aligned}
& \frac{1}{2}\left(f\left(\left(\mu_{l}^{-1}(\alpha)\right)^{p},\left(v_{l}^{-1}(\alpha)\right)^{q}\right)+f\left(\left(\mu_{r}^{-1}(\alpha)\right)^{p},\left(v_{r}^{-1}(\alpha)\right)^{q}\right)\right)-f\left(E\left[|\xi|^{p}\right], E\left[|\eta|^{q}\right]\right) \\
& \quad \leq \frac{a}{2}\left(\left(\mu_{l}^{-1}(\alpha)\right)^{p}+\left(\mu_{r}^{-1}(\alpha)\right)^{p}\right)-a E\left[|\xi|^{p}\right]+\frac{b}{2}\left(\left(v_{l}^{-1}(\alpha)\right)^{q}+\left(v_{r}^{-1}(\alpha)\right)^{q}\right)-b E\left[|\eta|^{q}\right] .
\end{aligned}
$$

Taking integration with respect to $\alpha$ on $[0,1]$, we have

$$
E\left[f\left(|\xi|^{p},|\eta|^{q}\right)\right] \leq f\left(E\left[|\xi|^{p}\right], E\left[|\eta|^{q}\right]\right)
$$

The inequality is thus verified.

Theorem 7 (Minkowski inequality) Let $p$ be a real number such that $p \geq 1$, and let $\xi$ and $\eta$ be independent uncertain sets with regular membership functions. Then we have

$$
\sqrt[p]{E\left[|\xi+\eta|^{p}\right]} \leq \sqrt[p]{E\left[|\xi|^{p}\right]}+\sqrt[p]{E\left[|\eta|^{p}\right]}
$$

Proof Note that $f(x, y)=(\sqrt[p]{x}+\sqrt[p]{y})^{p}$ is a concave function on $\{(x, y) \mid x \geq 0, y \geq 0\}$. Thus for any point $\left(x_{0}, y_{0}\right)$ with $x_{0}>0$ and $y_{0}>0$, there exist two real numbers $a$ and $b$ such that

$$
f(x, y)-f\left(x_{0}, y_{0}\right) \leq a\left(x-x_{0}\right)+b\left(y-y_{0}\right), \quad \forall x \geq 0, y \geq 0 .
$$

Let $\mu$ and $v$ denote the regular membership functions of uncertain sets $\xi$ and $\eta$, respectively. Taking $x_{0}=E\left[|\xi|^{p}\right], y_{0}=E\left[|\eta|^{p}\right], x=\left(\mu_{l}^{-1}(\alpha)\right)^{p}$ and $y=\left(v_{l}^{-1}(\alpha)\right)^{p}$, we have

$$
\begin{aligned}
& f\left(\left(\mu_{l}^{-1}(\alpha)\right)^{p},\left(v_{l}^{-1}(\alpha)\right)^{p}\right)-f\left(E\left[|\xi|^{p}\right], E\left[|\eta|^{p}\right]\right) \\
& \quad \leq a\left(\left(\mu_{l}^{-1}(\alpha)\right)^{p}-E\left[|\xi|^{p}\right]\right)+b\left(\left(v_{l}^{-1}(\alpha)\right)^{p}-E\left[|\eta|^{p}\right]\right)
\end{aligned}
$$

for all $\alpha \in(0,1)$. Taking $x_{0}=E\left[|\xi|^{p}\right], y_{0}=E\left[|\eta|^{p}\right], x=\left(\mu_{r}^{-1}(\alpha)\right)^{p}$ and $y=\left(v_{r}^{-1}(\alpha)\right)^{p}$, we have

$$
\begin{aligned}
& f\left(\left(\mu_{r}^{-1}(\alpha)\right)^{p},\left(v_{r}^{-1}(\alpha)\right)^{p}\right)-f\left(E\left[|\xi|^{p}\right], E\left[|\eta|^{p}\right]\right) \\
& \quad \leq a\left(\left(\mu_{r}^{-1}(\alpha)\right)^{p}-E\left[|\xi|^{p}\right]\right)+b\left(\left(v_{r}^{-1}(\alpha)\right)^{p}-E\left[|\eta|^{p}\right]\right)
\end{aligned}
$$

for all $\alpha \in(0,1)$. By the inequalities (5) and (6), we have

$$
\begin{aligned}
& \frac{1}{2}\left(f\left(\left(\mu_{l}^{-1}(\alpha)\right)^{p},\left(v_{l}^{-1}(\alpha)\right)^{p}\right)+f\left(\left(\mu_{r}^{-1}(\alpha)\right)^{p},\left(v_{r}^{-1}(\alpha)\right)^{p}\right)\right)-f\left(E\left[|\xi|^{p}\right], E\left[|\eta|^{p}\right]\right) \\
& \quad \leq \frac{a}{2}\left(\left(\mu_{l}^{-1}(\alpha)\right)^{p}+\left(\mu_{r}^{-1}(\alpha)\right)^{p}\right)-a E\left[|\xi|^{p}\right]+\frac{b}{2}\left(\left(v_{l}^{-1}(\alpha)\right)^{p}+\left(v_{r}^{-1}(\alpha)\right)^{p}\right)-b E\left[|\eta|^{p}\right] .
\end{aligned}
$$

Taking integration with respect to $\alpha$ on $[0,1]$, we have

$$
E\left[f\left(|\xi|^{p},|\eta|^{p}\right)\right] \leq f\left(E\left[|\xi|^{p}\right], E\left[|\eta|^{p}\right]\right)
$$

The inequality is thus verified. 


\section{Applications to knowledge representation}

In risk evaluation problems, representing incomplete knowledge plays a crucial role. So far, various kinds of knowledge have been considered such as expert opinions and poor statistical information, and different approaches have been proposed for representing the knowledge based on probability theory (Walley [28]), possibility theory (Dubois and Prade $[29,30])$, or the combination of these two theories (Baudrit and Dubois [31]). Inspired by Baudrit and Dubois [31], this section will give an application of the inequalities in the area of representing incomplete uncertain knowledge.

Let $(\Gamma, \mathcal{L}, \mathcal{M})$ be an uncertainty space, and $\xi$ be an uncertain set on $(\Gamma, \mathcal{L}, \mathcal{M})$. However, only partly information of $\xi$ is known due to technological or financial constraints, for example its expected value $E[\xi]$ and variance $V[\xi]$. This section aims at providing a nested family $\mathcal{A}(\xi)$ around $\xi$, which contains and represents the incomplete knowledge about $\xi$.

The Chebyshev inequality can be written as

$$
\mathcal{M}\{|\xi-E[\xi]| \leq x \sqrt{V[\xi]}\} \geq 1-\frac{1}{x^{2}}
$$

where $x>1$ is a real number. Then

$$
\mathcal{M}\{\xi \subset[E[\xi]-x \sqrt{V[\xi]}, E[\xi]+x \sqrt{V[\xi]}]\} \geq 1-\frac{1}{x^{2}} .
$$

Assume $\xi$ has a membership function $\mu$. It follows from the definition of membership function that

$$
\begin{aligned}
& \mathcal{M}\{\{\xi \subset[E[\xi]-x \sqrt{V[\xi]}, E[\xi]+x \sqrt{V[\xi]}]\} \\
& \quad=1-\mu(E[\xi]-x \sqrt{V[\xi]}) \vee \mu(E[\xi]+x \sqrt{V[\xi]}) .
\end{aligned}
$$

Then we have

$$
\mu(E[\xi]-x \sqrt{V[\xi]}) \vee \mu(E[\xi]+x \sqrt{V[\xi]}) \leq \frac{1}{x^{2}} .
$$

As a result, the nested family $\mathcal{A}(\xi)$ is

$$
\mathcal{A}(\xi)=\left\{\eta \mid \mu_{\eta}(E[\xi]-x \sqrt{V[\xi]}) \vee \mu_{\eta}(E[\xi]+x \sqrt{V[\xi]}) \leq \frac{1}{x^{2}}\right\}
$$

where $\mu_{\eta}$ is the membership function of the uncertain set $\eta$. Writing in the form of the inverse membership function, we have

$$
\mathcal{A}(\xi)=\left\{\eta \mid \mu_{\eta}^{-1}(\alpha) \subset\left[E[\xi]-\sqrt{\frac{V[\xi]}{\alpha}}, E[\xi]+\sqrt{\frac{V[\xi]}{\alpha}}\right], \alpha \in(0,1]\right\} .
$$

\section{Conclusions}

In this paper, we proved some inequalities in the framework of uncertain set theory including the Markov inequality, the Chebyshev inequality, the Jensen inequality, the Hölder inequality, and the Minkowski inequality. These inequalities were applied to the area of representing incomplete uncertain knowledge. 


\section{Competing interests}

The authors declare that they have no competing interests.

\section{Authors' contributions}

This work was carried out in collaboration between all authors. All authors read and approved the final manuscript.

\section{Author details}

${ }^{1}$ School of Information Engineering, Shandong Youth University of Political Science, Jinan, 250103, China. ${ }^{2}$ Key Laboratory of Information Security and Intelligent Control in Universities of Shandong, Jinan, 250103, China. ${ }^{3}$ School of Information, Renmin University of China, Beijing, 100872, China.

\section{Acknowledgements}

The authors are thankful to the worthy referees for their useful suggestions. This work was supported by National Natural Science Foundation of China (Grant No. 61074193 and No. 63174082)

Received: 18 January 2014 Accepted: 16 April 2014 Published: 07 May 2014

\section{References}

1. Liu, B: Why is there a need for uncertainty theory? J. Uncertain Syst. 6(1), 3-10 (2012)

2. Zadeh, LA: Fuzzy sets. Inf. Control 8, 338-353 (1965)

3. Zadeh, LA: Fuzzy sets as the basis for a theory of possibility. Fuzzy Sets Syst. 1(1), 3-28 (1978)

4. Dempster, AP: Upper and lower probabilities induced by a multivalued mapping. Ann. Math. Stat. 38(2), 325-339 (1967)

5. Shafer, G: A Mathematical Theory of Evidence. Princeton University Press, Princeton (1976)

6. Kahneman, D, Tversky, A: Prospect theory: an analysis of decision under risk. Econometrica 47(2), $263-292$ (1979)

7. Pawlak, Z: Rough sets. Int. J. Parallel Program. 11(5), 341-356 (1982)

8. Liu, B: Uncertainty Theory, 2nd edn. Springer, Berlin (2007)

9. Liu, B: Uncertainty Theory: A Branch of Mathematics for Modeling Human Uncertainty. Springer, Berlin (2010)

10. Gao, X: Some properties of continuous uncertain measure. Int. J. Uncertain. Fuzziness Knowl.-Based Syst. 17(3), 419-426 (2009)

11. You, C: Some convergence theorems of uncertain sequences. Math. Comput. Model. 49(3-4), $482-487$ (2009)

12. Liu, YH, Ha, M: Expected value of function of uncertain variables. J. Uncertain Syst. 4(3), 181-186 (2010)

13. Chen, X, Dai, W: Maximum entropy principle for uncertain variables. Int. J. Fuzzy Syst. 13(3), 232-236 (2011)

14. Liu, B: Uncertain set theory and uncertain inference rule with application to uncertain control. J. Uncertain Syst. 4(2), 83-98 (2010)

15. Gao, X, Gao, Y, Ralescu, DA: On Liu's inference rule for uncertain systems. Int. J. Uncertain. Fuzziness Knowl.-Based Syst. 18(1), 1-11 (2010)

16. Peng, Z, Chen, X: Uncertain systems are universal approximators. http://orsc.edu.cn/online/100110.pdf

17. Gao, Y: Uncertain inference control for balancing an inverted pendulum. Fuzzy Optim. Decis. Mak. 11(4), 481-492 (2012)

18. Liu, B: Uncertain logic for modeling human language. J. Uncertain Syst. 5(1), 3-20 (2011)

19. Liu, B: Membership functions and operational law of uncertain sets. Fuzzy Optim. Decis. Mak. 11(4), 387-410 (2012)

20. Yao, K: Entropy operator for membership function of uncertain set. http://orsc.edu.cn/online/120313.pdf

21. Wang, X, Ha, M: Quadratic entropy of uncertain sets. Fuzzy Optim. Decis. Mak. 12(1), 99-109 (2013)

22. Liu, B: Some research problems in uncertainty theory. J. Uncertain Syst. 3(1), 3-10 (2009)

23. Liu, B: Theory and Practice of Uncertain Programming, 2nd edn. Springer, Berlin (2009)

24. Yao, K: Uncertain calculus with renewal process. Fuzzy Optim. Decis. Mak. 11(3), 285-297 (2012)

25. Liu, B: Toward uncertain finance theory. J. Uncertain. Anal. Appl. 1, Article ID 1 (2013)

26. Yao, K: No-arbitrage determinant theorems on mean-reverting stock model in uncertain market. Knowl.-Based Syst. 35, 259-263 (2012)

27. Liu, B: A new definition of independence of uncertain sets. Fuzzy Optim. Decis. Mak. 12(4)، 451-461 (2013)

28. Walley, P: Statistical Reasoning with Imprecise Probabilities. Chapman \& Hall, London (1991)

29. Dubois, D, Prade, H: The mean value of a fuzzy number. Fuzzy Sets Syst. 24(3), 279-300 (1987)

30. Dubois, D, Prade, H: When upper probabilities are possibility measure. Fuzzy Sets Syst. 49(1), 65-74 (1992)

31. Baudrit, C, Dubois, D: Practical representations of incomplete probabilistic knowledge. Comput. Stat. Data Anal. 51, 86-108 (2006)

10.1186/1029-242X-2014-169

Cite this article as: Ning and Huang: Inequalities of uncertain set with its applications. Journal of Inequalities and Applications 2014, 2014:169 